\title{
EFFECTS OF ALLOPURINOL ON IRON METABOLISM IN MAN
}

\author{
BY
}

\author{
B. T. EMMERSON
}

From the Princess Alexandra Hospital, University of Queensland, Brisbane, Australia

We have treated only eight patients with allopurinol, selected because their hyperuricaemia and gout were uncontrollable by standard measures, and because they were prepared for detailed and prolonged study. In view of the theoretical possibility that inhibition of xanthine oxidase might also have an effect in iron metabolism (Green and Mazur, 1957; Mazur, Green, Saha, and Carleton, 1958; Mazur and Carleton, 1965) and the increase in the hepatic content of iron in rats given allopurinol (Powell and Emmerson, 1966), we were especially interested in any effects which allopurinol might have on iron metabolism or on haemopoiesis in these patients.

The first aspect of interest concerns the reticulocyte counts (Fig. 1) which are expressed as a percentage of the erythrocytes, the upper limit of normal being 2 per cent. The patient with the

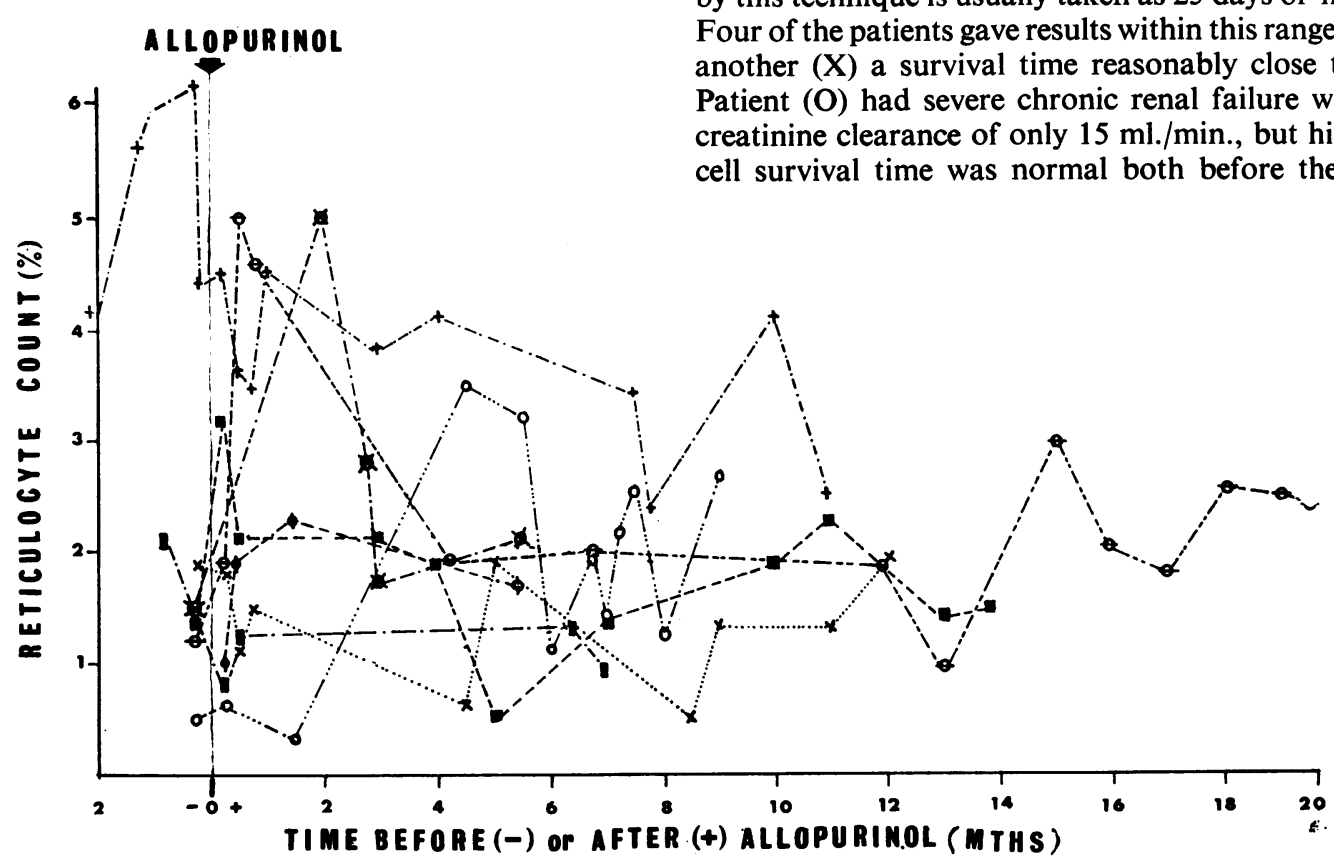

Fig. 1.-Relationship between reticulocyte count and duration of allopurinol therapy. persistently high reticulocyte count (marked + ) ha@ a myeloproliferative disorder with hypersplenism and a persistent reticulocytosis both before and aftep allopurinol therapy. If his results are disregardef it will be more clearly seen that some patients with normal reticulocyte counts before treatment di\& develop a variable reticulocytosis during treatment with allopurinol, especially in the first few months of treatment, but that, in general, these changes weres not sustained.

In view of this reticulocytosis, the next aspeco which was investigated was the red cell surviva 9 time, measured by the ${ }^{51}$ chromium labelling technique (Veall and Vetter, 1958). The results if relation to months of allopurinol therapy are show in Fig. 2 (opposite).

The normal red cell survival time which is shogrw by this technique is usually taken as 25 days or me. Four of the patients gave results within this range another (X) a survival time reasonably close to it. Patient (O) had severe chronic renal failure with creatinine clearance of only $15 \mathrm{ml} . / \mathrm{min}$., but his reg cell survival time was normal both before therap $\overline{\bar{y}}$ 


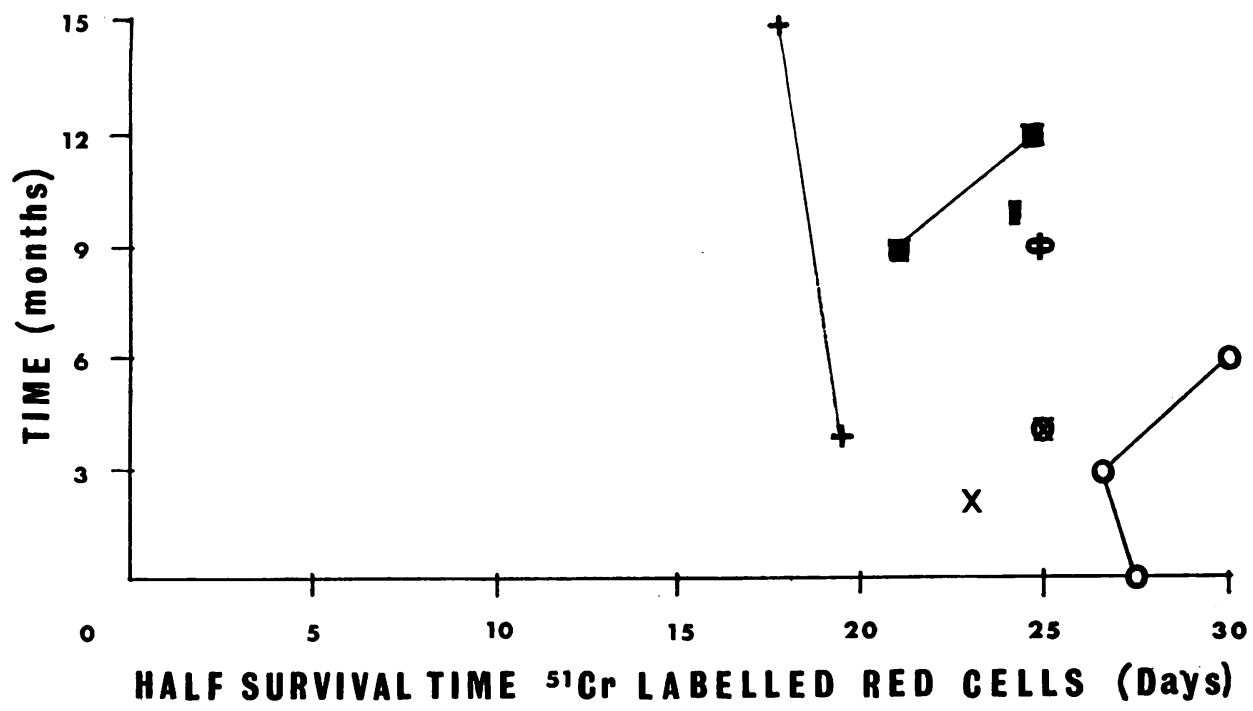

Fig. 2.-Relationship between red cell survival time and duration of allopurinol therapy.

and during a period of increased anaemia 6 months after beginning therapy. This shortened survival time of 21 days in patient $(\square)$ was normal when repeated 3 months later. Patient $(+)$, as in Fig. 1 the patient with a myeloproliferative disorder, had hypersplenism with a high splenic uptake of radio-activity. His red cell survival time shortened by only one day during 12 months of allopurinol therapy. In general, therefore, our results did not suggest any shortening of the red cell survival time with allopurinol therapy.

From the time of our first interest in the question of iron metabolism, we followed the serum iron values in patients under treatment (Fig. 3). In most patients, allowing for the wide fluctuations known to occur in the serum iron concentration from day to day and week to week, the serum iron concentrations obtained were probably not abnormal. Some



Fig. 3.-Relationship between serum iron concentrations and duration of allopurinol therapy. 
patients $(\theta$ and $\theta)$ showed occasional abnormal readings after 12 months of treatment, but one patient (I) showed frequent results of over $200 \mu \mathrm{g}$./ $100 \mathrm{ml}$. during a period of 12 months, only two out of eight observations being within the normal range. This patient has severe hyperlipaemia and coronary artery disease and was receiving anticoagulants both before and since allopurinol was commenced. From 6 months after allopurinol therapy was begun, he has also been receiving chlorphenoxyisobutyrate ("Atromid"). Though there is no recognized effect of this drug on iron metabolism, its use does introduce a complicating factor in assessing the significance of his serum iron concentrations. The patient also had a high serum iron-binding capacity of $448 \mu \mathrm{g} . / 100 \mathrm{ml}$. at a time when the serum iron was $210 \mu \mathrm{g} . / 100 \mathrm{ml}$.

In summarizing this aspect in these patients, therefore, the serum iron concentrations have been within the normal range except for isolated readings. The one patient with a consistently high serum iron concentration demonstrates an unusual fluctuation in these levels and is also receiving other drugs.

Because of difficulty in interpreting the significance of these serum iron results, a test of the extent of iron stores was undertaken using a chelating agent; we

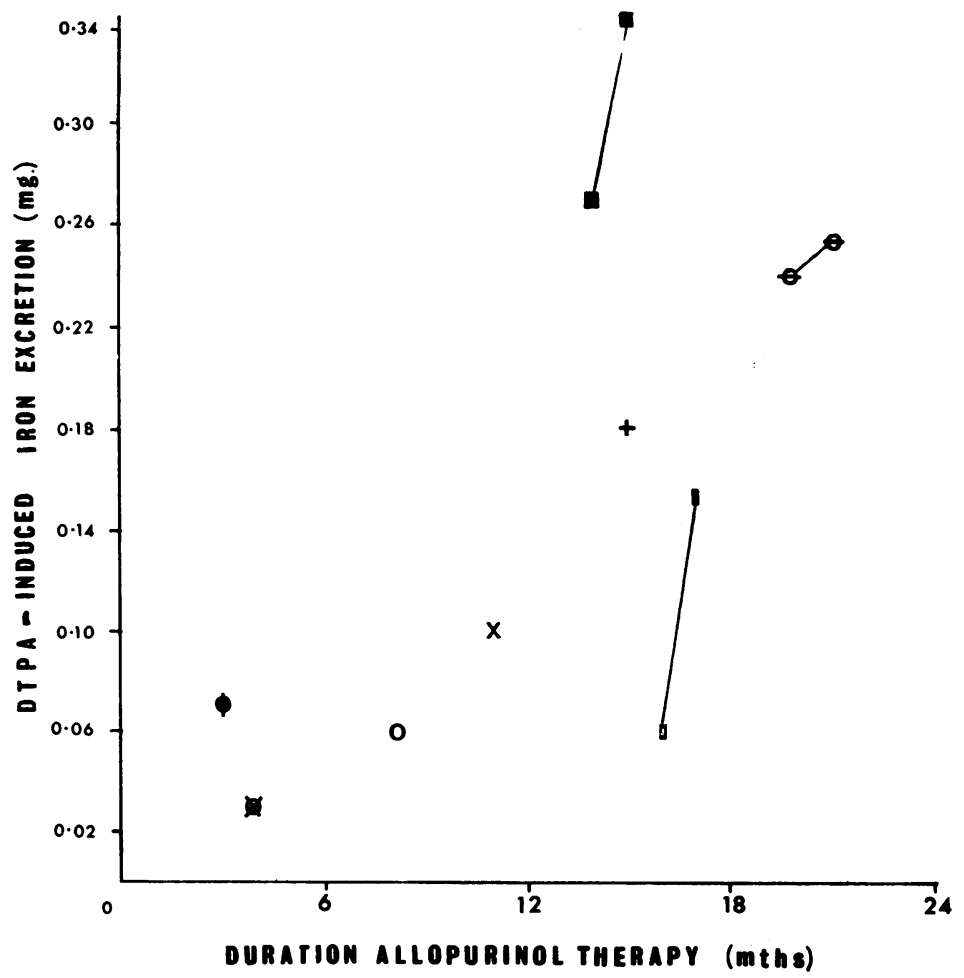

Fig. 4.-Relationship between iron excretion following DTPA and duration of allopurinol therapy. measured the urinary excretion of iron in the 6 houा s after the beginning of a 1-hour infusion of $1 \mathrm{cs}$. di-ethylene-triamine-penta-acetate (DTPA). The normal range has recently been established in the Brisbane population by Powell (1965) as 0.16 g $0.06 \mathrm{mg}$. with skewing of the distribution towares the lower range. The resulting iron excretions plotted against the duration of allopurinol thera柦y (Fig. 4). It may be seen that, in general, the irgn excretion after DTPA tends to rise with increasing duration of allopurinol treatment. The values of 0.24 and 0.27 (shown by $\theta$ and $\theta$ ), although within the normal range, would rarely occur in a group@f eight subjects within the normal population, af $d$ were regarded with suspicion. The patient with the persistently high serum iron (I) had a very 1 ow iron excretion after DTPA. One month later, the iron excretions after DTPA were repeated in threeonf the patients, the second result being joined to the first by a line. None had fallen and one $(\theta)$ higd risen beyond the normal range.

In view of this finding, and after full discussign with the patients involved, liver biopsies were carried out on the two patients $(\theta$ and $\theta)$ with the high DTPA-induced iron excretions. However, 
deposition in the liver using specific iron stains was not increased beyond that which would be found in the normal Brisbane population. Thus, in patients treated with allopurinol for up to 20 months, we found no unequivocal evidence of increased iron storage. However, as 20 months is a relatively short period in the life span of man and as it is possible that iron deposition may take even longer than this to develop, it seems reasonable to continue observation of the serum iron concentration and of the DTPA-induced iron excretion in patients on prolonged allopurinol therapy. However, it is clear that the slower the deposition, the less significant does any iron accumulation become.

In view of the apparent role of xanthine oxidase in the absorption of iron from the alimentary tract, I would like to add that Prof. D. J. Deller of Adelaide, South Australia, has studied the effect of premedication with allopurinol on the absorption of a tracer dose of iron in man. With allopurinol on the 2 days preceding and on the day of administration of the tracer dose of ${ }^{59} \mathrm{Fe}$, he has demonstrated no sig- nificant effect on the alimentary absorption of iron. $\mathrm{He}$ is proceeding to further studies using larger doses of allopurinol for a longer time.

Finally, I should like to make the point that, if allopurinol does have any effect on iron metabolism, such an effect may be seen in only some of the patients so treated, and not necessarily in all. Of the three recorded patients with xanthinuria, only one has haemochromatosis (Ayvazian, 1964). Although our investigations have not, at this stage, clearly demonstrated any deleterious effect of allopurinol on iron metabolism, we feel that it may yet be prudent to continue these observations for a longer period.

I should like to acknowledge the ready assistance provided in this investigation by the Divisions of Biochemistry of the Royal Brisbane Hospital and the Princess Alexandra Hospital, especially that of Mr M. J. Thomas who undertook the urinary iron estimations. I am also indebted to Dr. M. D. Innes of the Division of Haematology of the Princess Alexandra Hospital for the red cell survival studies. The DTPA was generously supplied by Dr. W. Inglis of Geigy Pharmaceuticals (Australia). 